\title{
Comparative study of visual inspection of the cervix by $3 \%$ acetic acid (VIA) versus Pap smear by Bethesda method in sexually active women aged 25-50 years as an equally or more effective cervical cancer screening method in a low resource setup
}

\author{
Mohit Rajendra Saraogi ${ }^{1}$, Alka Gupta ${ }^{2}$
}

\begin{abstract}
${ }^{1}$ Department of Obstetrics \& Gynaecology, Cama \& Albless Hospital, Grant Government Medical College \& Sir J. J. Group of Hospitals, Mumbai, Maharashtra, India

${ }^{2}$ Department of Obstetrics \& Gynaecology, Seth G S Medical College \& KEM Hospital, Mumbai, Maharashtra, India
\end{abstract}

Received: 26 July 2014

Accepted: 8 August 2014

\section{*Correspondence:}

Dr. Mohit Rajendra Saraogi,

E-mail: mohitsaraogi85@gmail.com

(C) 2014 Saraogi MR et al. This is an open-access article distributed under the terms of the Creative Commons Attribution Non-Commercial License, which permits unrestricted non-commercial use, distribution, and reproduction in any medium, provided the original work is properly cited.

\begin{abstract}
Background: Cervical cancer is the most common cancer in Indian women and is a preventable cancer. Pap smear being an expensive screening test, increased emphasis is being laid on the development of a reliable and cost effective screening method for cervical cancer. This study aims at early detection of cervical dysplastic lesions using a simple and cost-effective screening test like visual inspection of cervix with 3\% acetic acid (VIA) and comparing its diagnostic efficacy with the more expensive Pap screening by Bethesda method.

Methods: Ours was a prospective study carried out on a 100 sexually active women aged 25-50 years, coming to our OPD. The women were subjected to both a VIA and Pap smear. All Pap and VIA positive women were subjected to a cervical biopsy, whose histopathological report was taken as the gold standard.

Results: In our study the sensitivity of VIA was more than that of cytology (100\% versus $66.67 \%)$ but the specificity was significantly lesser (47.83\% compared to the $73.91 \%$ ). The negative predictive value of VIA was comparable with Pap smear (100\% \& 85\% respectively) as was the positive predictive value $(42.86 \% \& 50 \%)$. However the diagnostic accuracy of VIA was lower than that of Pap smear (66.67\% \& 81.25\%) in our study.

Conclusions: In this study VIA was found to have efficacy comparable to Pap smear in screening cervical cancer. Thus we recommend that VIA could be used as an alternative screening tool to detect early cervical dysplasia especially in poor resource settings.
\end{abstract}

Keywords: Pap smear, Bethesda, VIA, Cervical cancer, Screening tests

\section{INTRODUCTION}

Cancer of the cervix is the most common cancer in Indian women. ${ }^{1}$ The estimated new cervical cancer cases per year is 493000 globally of which $79 \%$ occur in the developing countries. ${ }^{1}$ Cervical cancer still remains a significant problem in India, as India alone accounts for 100000 new cases of cervical cancer every year and over 80000 deaths every year. ${ }^{2}$
Cervical cancer is essentially a preventable cancer as it has a long premalignant phase, during which if it is detected and treated, advanced disease \& potential mortality can be prevented. ${ }^{3}$ Unfortunately more than $80 \%$ of the cases are diagnosed at an advanced clinical stage when the five year survival rates are less than $40 \%{ }^{4}$ Keeping this in mind, increased emphasis is being laid on the early detection of cervical intraepithelial changes, and hence the development of a reliable and cost effective screening method for cervical cancer. 
For years Pap smear has remained the most common screening test for cervical cancer. However many developing countries do not have ample resources to implement cytology-based prevention programs. The screening coverage of Pap smear in India is only 2.6-5\% as compared to $50 \%$ in Singapore and $68-84 \%$ in developing countries - where there exists a successful cervical cancer screening program. ${ }^{5-7}$

Hence low cost alternatives to Pap smear, such as Visual Inspection of cervix using Acetic acid (VIA), \& Visual Inspection of cervix using Lugols Iodine (VILI) have recently emerged.

Our study aims at early detection of cervical dysplastic lesions using a simple and cost-effective method like Visual Inspection of cervix with 3\% Acetic acid (VIA) and comparing its diagnostic efficacy with the relatively more expensive and time tested method of cytology (Pap smear).

\section{METHODS}

This was a prospective comparative study carried out in a group of 100 sexually active women aged 25-50 years presenting to the gynaecology OPD of KEM hospital, Mumbai with various complaints such as post coital bleeding, leucorrhoea, intermenstrual bleeding or cervical erosion/cervical irregularities on examination. The patients were subjected to Pap smear examination by Bethesda method, followed by visual inspection of the cervix by $3 \%$ acetic acid. This study was carried out over a period of 15 months ranging from July 2011 to October 2012, after approval from the institutional ethics committee. All the women had no preceding factors prohibiting Pap smear examination or VIA.

The following groups of women were excluded from our study -

- Women undergone hysterectomy

- Women in the menstrual phase of their cycle

- Pregnant women

- Women less than 25 years or more than 50 years of age

- Women with vaginal medications, vaginal contraceptives or douches

- Women in puerperium

- Women with white discharge of infective etiology

All patients presenting with acetowhite lesions on application of acetic acid were recorded as VIA positive and their findings were documented with the help of a cervicogram. Patients not having acetowhite lesions were documented to be VIA negative.

All patients with Pap smear findings of ASCUS and above were recorded as Pap positive for malignancy. All VIA or Pap positive patients were subjected to a cervical biopsy, the histopathological findings of which were taken as gold standard in this study.

\section{RESULTS}

VIA \& Pap smear were performed on 100 women presenting to the OPD. Twenty one women out of the 100 (21\%) had acetowhite lesions \& 12 women (12\%) were Pap positive for malignancy. Seven women who were Pap positive were also VIA positive. Hence a total of 26 women were subjected to a cervical biopsy.

Table 1: Comparison of VIA and Pap findings.

\begin{tabular}{|llll|}
\hline \multirow{2}{*}{ VIA } & \multicolumn{2}{l}{ Pap smear } & \multicolumn{1}{c}{ Total } \\
\cline { 1 - 3 } Positive & 7 & Negative & \\
\hline Negative & 5 & 14 & 21 \\
\hline Total & 12 & 88 & 79 \\
\hline
\end{tabular}

The twelve pap positive cases (12\%) had various grades of cervical dysplasia on cytology reports, of which high grade lesions (HSIL) were seen in 6 cases (6\%). 2 cases (2\%) had ASCUS on cytology \& 4 cases $(4 \%)$ had LSIL on pap smear.

Out of the 26 cases subjected to a cervical biopsy, no malignancy was found in 17 cases $(65.38 \%)$. Two cases (7.6\%) had severe dysplasia, 1 case $(3.8 \%)$ had CIN III, 1 case had squamous cell carcinoma in situ, 4 cases $(15.2 \%)$ cases had invasive squamous cell carcinoma \& 1 case $(3.8 \%)$ had adenosquamous carcinoma.

Out of the 26 cases which underwent a cervical biopsy, cervical cancer was detected in 9 women. All of these 9 women $(100 \%)$ were VIA positive while only 6 women out of these $9(66.67 \%)$ were Pap positive for malignancy. As a screening test, with cervical biopsy as a gold standard, the sensitivity of Pap smear was $66.67 \%$ and the specificity was $73.91 \%$. The positive predictive value of Pap smear was $50 \%$ \& the negative predictive value was $85 \%$ with a diagnostic accuracy of $71.88 \%$

In comparison the sensitivity and specificity of VIA was $100 \%$ and $47.83 \%$, respectively. The positive predictive value of VIA was $42.86 \%$ \& the negative predictive value was $100 \%$ with a diagnostic accuracy of $62.5 \%$.

Table 2: Comparison of sensitivity and specificity.

\begin{tabular}{|lll|}
\hline & Pap smear & VIA \\
\hline Sensitivity & $66.67 \%$ & $100 \%$ \\
\hline Specificity & $73.91 \%$ & $47.83 \%$ \\
\hline Positive predictive value & $50 \%$ & $42.86 \%$ \\
\hline Negative predictive value & $85 \%$ & $100 \%$ \\
\hline Diagnostic accuracy & $71.88 \%$ & $62.50 \%$ \\
\hline
\end{tabular}

When both VIA and cytology were combined together the negative predictive value was found to be $100 \%$. The 
sensitivity was $100 \%$, but the specificity was found to be $26.09 \%$. The positive predictive value in this group was found to be $34.62 \%$.

\section{DISCUSSION}

Our study results showed that sensitivity of VIA was more than that of cytology $(100 \%$ versus $66.67 \%)$ but the specificity was significantly lesser $(47.83 \%$ compared to the $73.91 \%$ ). Since sensitivity is the primary criteria for a screening test in places having a high prevalence of a disease, VIA can definitely be more helpful in low resource settings as compared to cytology as an initial screening test for cervical cancer. Patients presenting with acetowhite lesions can then be subjected to further evaluation.

It is interesting to note that in our study VIA was able to detect all the 9 cases of cervical cancer unlike pap which could detect only 6. Keeping the high prevalence of cervical cancer in India, this would make VIA a better screening test by itself than Pap smear.

Out of the 21 VIA positive cases, 7 cases had an abnormal Pap smear report also.

6 out of $7(85.71 \%)$ of these cases had cervical cancer on biopsy. This indicates that cases having both, an abnormal pap report and acetowhite lesions on VIA, have an extremely high probability of cervical cancer \& will require more aggressive management.

The negative predictive value of VIA was comparable with Pap smear $(100 \%$ \& $85 \%$ respectively) as was the positive predictive value $(42.86 \% \& 50 \%)$. However the diagnostic accuracy of VIA was marginally lower than that of Pap smear $(66.67 \% \& 81.25 \%)$ in our study.

The Table 3 below compares our findings with other studies comparing the sensitivity and specificity of VIA and cytology:

Table 3: Comparison of our findings with other studies comparing the sensitivity and specificity of VIA and cytology.

\begin{tabular}{|llllll|}
\hline Author & VIA sensitivity & VIA specificity & Pap sensitivity & Pap specificity & Cut-off level \\
\hline Megavand et al. (1996) & 0.64 & 0.98 & 0.62 & 0.89 & - \\
\hline Denny et al. (2000) & 0.67 & 0.83 & 0.75 & 0.90 & - \\
\hline Quereshi et al. (2010) & 0.55 & 0.71 & 0.20 & 0.98 & CIN I+ \\
\hline Present study (2012) & 1.00 & 0.48 & 0.66 & 0.74 & - \\
\hline & & & & & \\
\hline Sankaranarayanan et al. (2003) & 0.82 & 0.78 & 0.81 & 0.87 & CIN II+ \\
\hline Zimbabwe project (1999) & 0.77 & 0.65 & NA & NA & CIN II+ \\
\hline
\end{tabular}

Our study shows that keeping cervical biopsies in mind, 21 cases of VIA positive patients would have required a cervical biopsy, whereas only 12 cases of Pap positive women would have required a cervical biopsy. Hence the incidence of cervical biopsy is almost $200 \%$ more with VIA as compared with Pap smear in our study. Logistically, the average cost of a pap smear in most laboratories in Mumbai ranges from 600 to a 1000 rupees, while surprisingly the cost of a histopathological reporting of a cervical biopsy report was cheaper and ranged from 600-700 rupees. The cost of HPV DNA testing costs around rupees 2200 in most laboratories and is not routinely done in the government setup. The cost of acetic acid by itself in VIA comes to less than 5 rupees per patient. Hence even with the increased incidence of cervical biopsy with VIA, the overall cost of VIA turns out to be significantly cheaper than that of Pap smear. Also, as cervical biopsy can be performed as an outpatient procedure, the procedure itself does not provide a drain on the hospital resources \& does not significantly add to the patients costs.
Recent recommendations on cervical cancer screening by American Cancer Society, make no mention of VIA, but HPV screening has been added. They do not keep in mind that the per patient expenditure in such a screening programme would be well beyond the financial capability of most developing countries including India. The need of the hour in developing countries is to develop a cheap, efficacious \& viable screening programme with widespread coverage.

And Inclusion of VIA in these screening programmes may just be the solution to this problem \& definitely requires consideration.

\section{CONCLUSIONS}

VIA in this study was found to have efficacy comparable to Pap smear in screening cervical dysplastic changes. Thus we recommend that visual inspection of cervix by acetic acid could be used as an alternative screening tool to detect early cervical pre-cancer especially in poor resource settings. These methods do not require advanced 
technology and they may be performed by paramedics after appropriate training. Nurses, auxiliary nurses \& midwives can be easily trained to insert a speculum \& examine the cervix visually, before and after application of acetic acid. They then could be instructed to further refer the patients presenting with abnormal acetowhite lesions on application of acetic acid to the cervix, to higher centres for further evaluation. Screening by VIA has the potential of making feasible the screening of vast number of women in developing parts of the world for cervical cancer, \& hence reducing the global burden of morbidity \& mortality due to cervical cancer.

This study throws insight into the prevailing conditions in rural India. We expect more severe outcomes in the actual community as home deliveries by untrained birth attendants and unattended deliveries are common. Rheumatic heart disease continues to be the leading cause of cardiac illness and is associated with unpleasant outcomes. In the second trimester cardiac demands begin to approach their zenith. This is a crucial period and chances of cardiac failure are escalated. It is important to educate masses about these concepts and implement screening camps to detect young women with cardiac defects.

The need for providing doorstep services to people in rural India and training rural health workers to motivate every pregnant woman to seek expert care during her pregnancy cannot be overemphasized and must be undertaken with utmost priority.

\section{Funding: No funding sources}

Conflict of interest: None declared

Ethical approval: The study was approved by the institutional ethics committee

\section{REFERENCES}

1. Alliance for Cervical Cancer Prevention. Preventing cervical cancer worldwide. In: ACCP, eds. ACCP Document. Washington: PATH Alliance Coordinating Agency; 2004: 1-24.

2. Jugal Kishore, Vishal Mundra, Indu Grewal. Perception and use of Pap smear among medical personnel in New Delhi, India. Health Popul Persp Issues. 2009;32(3):141-7.

3. Awasthy S, Quereshi MA, Kurian B, Leelamoni K. Cervical cancer screening: current knowledge \& practice among women in a rural population of Kerala, India. Indian J Med Res. 2012 Aug;136:20510 .

4. Surendra S. Shastri, Ketayun Dinshaw, Geetanjali Amin, Smriti Goswamy, Sharmila Patil, Roshini Chinoy, et al. Concurrent evaluation of visual, cytological and HPV testing as screening methods for the early detection of cervical neoplasia in Mumbai, India. Bull WHO. 2005 Mar;83(3):186-94.

5. Nor HO, Matejka R. Challenges to cervical cancer screening in a developing country: the case of Malaysia. Asia Pacific J Cancer Prev. 2009;10:74752.

6. WHO. World Health Survey, 2003. Available at: http://www.who.int/healthinfo/survey/en/.

7. Gakidou E, Nordhagen S, Obermeyer Z. Coverage of cervical cancer screening in 57 countries: low average levels and large inequalities. PLoS Med. 2008;5:e132.

DOI: $10.5455 / 2320-1770 . \mathrm{ijr} \operatorname{cog} 20140964$

Cite this article as: Saraogi MR, Gupta A. Comparative study of visual inspection of the cervix by $3 \%$ acetic acid (VIA) versus Pap smear by Bethesda method in sexually active women aged 25-50 years as an equally or more effective cervical cancer screening method in a low resource setup. Int J Reprod Contracept Obstet Gynecol 2014;3:688-91. 